\title{
Massive physical and dynamical characterization of asteroids
}

\author{
William Thuillot ${ }^{1}$, Jerôme Berthier ${ }^{1}$, Armand Sarkissian ${ }^{2}$, \\ Areg M. Mickaelian ${ }^{3}$, Lena A. Sargsyan ${ }^{3}$, Jesus Iglesias ${ }^{1}$, \\ Valery Lainey $^{1}$, Mirel Birlan ${ }^{1}$ and Guy Simon ${ }^{4}$ \\ ${ }^{1}$ IMCCE, Paris Observatory, 77 avenue Denfert Rochereau, Paris, France \\ email: thuillot,berthier@imcce.fr \\ ${ }^{2}$ Service d'aéronomie, Institut Pierre Simon Laplace, France \\ ${ }^{3}$ Byurakan Astrophysical Observatory, Armenia \\ ${ }^{4}$ GEPI, Observatoire Paris-Site de Meudon, 5 Pl J. Janssen, F-92195 Meudon Cedex, France \\ email: guy.simon@obspm.fr
}

Numerous new Solar System objects, mainly asteroids, are still detected nowadays but their physical and dynamical characteristics remain not accurately determined, until dedicated observations and analysis are made. However, the Virtual Observatory is a perfect framework to search for this characterization by data mining. We are developing two studies for this goal by exploring the DENIS infrared and DFBS spectroscopic surveys. In order to scan the survey catalogues and to search for Solar System objects, we have developed a VO compliant workflow founded on the use of a recent VO tool labelled SkyBoT (Sky Bodies Tracker) (Berthier et al. 2005) and on the VizieR service provided by CDS. A public version of SkyBoT is implemented in the sky atlas Aladin since January 2006. It allows us to quickly get the precise coordinates of the asteroids, planets and natural satellites in any star field, provided we know its center, its size and the precise date of the exposure (between 1949 and 2009). Our data mining workflow performs cone search queries on SkyBoT and VizieR to extract all the known astronomical objects observed by the survey. Then the sources which are detected in the survey are correlated with the known objects to determine the matched and unmatched objects.

The DENIS survey has been performed from 1995 to 2000 on a $1 \mathrm{~m}$ telescope at La Silla, ESO. It leads to astrometric and $I, J, K$ ' photometric measurements. A previous work carried out by Baudrand et al. (2004) had already led to the detection of 2190 associations and the identification of 1931 asteroids by comparison to the ephemerides of only 9000 objects. We are carrying out a new exploration of this wide database by comparing now to the ephemerides of more than 340,000 asteroids. The DFBS (Digitized First Byurakan Spectroscopic Survey) has been performed from 1965 to 1980 on the $1 \mathrm{~m}$ Schmidt telescope of the Byurakan Observatory equipped with a $1^{\circ} .5$ prism objective (Mickaelian et al. 2005). It was mainly dedicated to galaxies study. More than 15,000 spectra of low resolution $(50 \AA)$ in the spectral range $3400-6900 \AA$ are present on each one of the 2180 plates $\left(4^{\circ} \mathrm{FOV}\right.$ ). This led to about 40 millions of spectra in which we will identify those of Solar System objects. This is the first work dedicated to the extraction of Solar System data from this data base.

\section{References}

Baudrand, A., Bec Borsenberger, A., \& Borsenberger, J. 2004, A 6 A, 423, 381

Berthier J., Vachier F., Thuillot W., et al. 2005, in: Proc. ADASS XV, ASP-CS, 351, 367

Mickaelian, A. M., Hagen, H.-J., Sargsyan, L. A., \& Mikayelyan, G. A 2005, in: CDS VizieR On-line Data Catalog: VI/116 